\title{
Confirmation de la présence de Stipa apertifolia subsp. longiglumis (H. Scholz) Vázquez \& Devesa (Poaceae) dans les Monts des Ksour (Aïn Sefra, Algérie)
}

\author{
Belkacem Gordo, Seghir Hadjadj-Aoul \\ Département de Biologie, Université d'Oran 1, Ahmed Benbella, 31100, Dz.
}

\section{Correspondencia}

B. Gordo

e-mail: kacimo.elgordo96@gmail.com

Recibido: 25 abril 2021

Aceptado: 15 junio 2021

Publicado on-line: 1 julio 2021

Editado por: Noelia Hidalgo Triana

\begin{abstract}
Résumé
Dans cette note, les auteurs confirment la présence de Stipa apertifolia subsp. longiglumis $(\mathrm{H}$. Scholz) Vázquez \& Devesa dans l'Atlas saharien occidental. De même, ils actualisent les connaissances sur son écologie, son statut de conservation et celui de son endémicité.
\end{abstract}

Mots clés: Stipa, conservation, chorologie, systématique, Nâama.

\begin{abstract}
Confirmation of the presence of Stipa apertifolia subsp. longiglumis $(H$. Scholz) Vázquez \& Devesa (Poaceae) in the Ksour Mountains (Aïn Sefra, Algérie)

In this note, the authors confirm the presence of Stipa apertifolia subsp. longiglumis $(\mathrm{H}$. Scholz) Vázquez \& Devesa in the Western Saharan Atlas. Likewise, they update knowledge on its ecology, its conservation status and that of its endemicity.
\end{abstract}

Keywords: Stipa, conservation, chorology, taxonomy, Nâama.
En Algérie, la famille des Poaceae est représentée par 456 taxons dont seize (16) seraient endémiques. Dans les Monts des Ksour, elle renferme un nombre important de taxons, variant entre 60 (Benaissa, 2019) et 53 (Gordo, 2021). Au sein de cette famille, c'est le genre Stipa qui semble le plus intéressant du fait de son rôle écologique et physionomique joué dans les formations des paysages steppiques. En effet, parmi les dix-neuf (19) taxons acceptés relevant de ce genre, deux (2) sont des endémiques algériennes (Stipa atlantica Smirn. et $S$. hoggarensis Chrtek \& Martinovsky) et quatre (4) taxons sont des endémiques algéro-marocaines. Ce sont: Stipa apertifolia Martinovsky subsp. apertifolia, S. barbata subsp. brevipila (Coss. \& Durieu) F.M. Vázquez \& Devesa, S. celakovskyi Martinovsky, S. maroccana H. Scholz (Dobignard \& Chatelain, 2010; African Plant Database, 2012).

La majeur partie de nos prospections botaniques, entamées en 2021, ont été consacrées à la prospection de la végétation de montagne. Outre l'aspect préforestier, le paysage végétal d'altitude se caractérise par une flore tellienne typiquement méditerranéenne (Maire, 1916). Ainsi, les Monts des Ksour présentent une orographie particulière: des altitudes dépassant les 2000 m dont le parc national du Djebel Aïssa qui culmine à $2236 \mathrm{~m}$ et une diversité d'expositions et d'habitats. Lors de la réalisation de nos relevés floristiques dans les escarpements gréseux altitudinaux qui sont difficilement accessibles, nous avons observé une poacée discrète qui semble «s'abriter» de la dent du bétail, notamment, la chèvre, un vrai fléau régional (Gordo \& Hadjadj-Aoul, 2019; Gordo, 2021). Pour identifier cette poacée, nous avons fait appel aux clés d'identification de la Flore de Quézel \& Santa (1962) et celle de Maire (1953). L'actualisation des combinaisons anciennes par le biais de l'African Plant Database (2021), nous a conduits à Stipa apertifolia subsp. Iongiglumis $(\mathrm{H}$. Scholz) Vázquez \& Devesa. Nous avons déposé un spécimen de la plante à l'Herbier du laboratoire de botanique de l'université Oran 1 A. Benbella sous le numéro SAM. 00166.

\section{Morphologie}

II s'agit d'une plante plus au moins robuste de 0,40 à $0,50 \mathrm{~m}$ dont les chaumes sont dressés, raides et vêtus de gaines foliaires à nœuds glabres avec un lemme à arrête de $20-30 \mathrm{~cm}$ de long, nue au dessus du genou et à poils très longs (Figure 1). Les gaines des feuilles âgées et/ou mortes sont jaunes sinon translucides. Les pédoncules sont épais et la ligule ovale lancéolée, glabre ou subglabre, plus ou moins lacérée. Le limbe condupliqué dressé est long de 25 à $30 \mathrm{~cm}$ et large de 0,7 à 0,9 mm (Maire, 1953; Quézel \& Santa, 1962; Vázquez \& Devesa 1996a; Fennane et al., 2014).

\section{Systématique et chorologie}

Bien qu'absente des listes floristiques des anciens botanistes qui ont exploré les Monts des Ksour tels que Hochreutiner (1904), Maire (1916), cette espèce figure néanmoins dans le catalogue floristique du Maroc de Sauvage et Vindt (1949). En effet, cette plante avait été décrite par Maire (1953) sous le binôme: Stipa pennata L. subsp. mediterranea (Trin. 
\& Rupr.) Asch. \& Gr., combinaison reprise plus tard par Quézel \& Santa (1962). D'après ces auteurs, ce taxon présente une répartition eurasiatique.

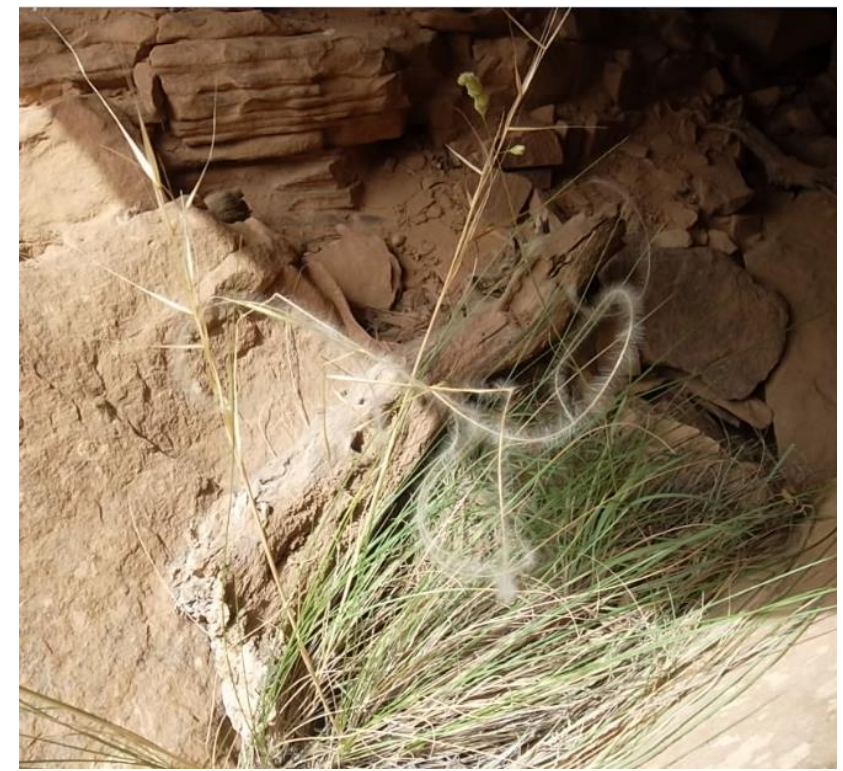

Figure 1. Stipa apertifolia subsp. Iongiglumis (H. Scholz) Vázquez \& Devesa dans son biotope (Dj. M'Zi, 2075 m; photo B. Gordo).

Figure 1. Stipa apertifolia subsp. longiglumis (H. Scholz) Vázquez \& Devesa in its biotope (M'Zi mountain, $2075 \mathrm{~m}$; photo B. Gordo).

En révisant le genre Stipa dans la Péninsule Ibérique, Vázquez \& Devesa (1996 b) sont arrivés à conclure que Stipa dasyvaginata Martinovsky est synonyme de Stipa apertifolia Martinovsky. Ainsi, c'est au sein de cette dernière espèce que Vázquez \& Devesa (1996 a) ont reconnu trois taxons, dont Stipa apertifolia subsp. longiglumis ( $\mathrm{H}$. Scholz) Vázquez \& Devesa. Ces auteurs se sont référés à I'holotype déposé dans l'Herbier de Berlin ("Algerien: NO. Djebel Megriss (N Sétif), ca. $1730 \mathrm{~m}$, Fels-und Weide-Iriften, 11.7.1983, H. Scholz \& G. Baillargeon 95"). Cela les avait amenés à limiter son aire de répartition au nord-est de l'Algérie. D'autre part, ce taxon a été considéré comme endémique marocain par Ibn Tattou \& Fennane (2009); Dobignard \& Chatelain (2010) et El Oualidi et al., (2012). Récemment, Fennane et al. (2014) n'ont retenu que l'espèce Stipa apertifolia. Cette dernière est endémique de l'Algérie, du Maroc et de la péninsule ibérique. Plus récemment, Fennane (2018) adopte une autre position et considère ce taxon comme endémique du Maroc. II convient par ailleurs de signaler qu'Aedo (2020) considère S. apertifolia comme faisant partie des synonymes de $S$. iberica Martinovský. Ainsi, la confirmation de la présence de ce taxon dans les Monts des Ksour se veut une contribution venant répondre à ces incertitudes d'ordre chorologique. En effet, cette nouvelle donnée étend plus à l'ouest la répartition connue du taxon en Algérie. Elle sert également à actualiser son statut d'endémisme.

\section{Habitat et écologie}

C'est en juillet 2018 que nous avons observé Stipa apertifolia subsp. longiglumis $(\mathrm{H}$. Scholz) Vázquez \& Devesa dans les escarpements gréseux du Djebel M'Zi en exposition nord-est à $2075 \mathrm{~m}$ d'altitude. Bien qu'elle soit peu pentue $(10 \%)$, la station de découverte semble être à l'abri du bétail, compte tenu de la nature rude et rocheuse de ces milieux rupestres dépourvus le plus souvent de sol. Le recouvrement de ce type de végétation avoisine $40 \%$. Les données stationnelles ainsi que les espèces figurent dans le relevé floristique unique contenant l'espèce (cf. infra) (Tableau 1).

Stipa apertifolia subsp. Iongiglumis est une hémicryptophyte à floraison estivale. Elle se comporte comme chasmophyte comme tout le cortège floristique propre à ces biotopes. Du fait de ses exigences altitudinales (températures hivernales $<-3^{\circ} \mathrm{C}$, pluviométrie annuelle $>400 \mathrm{~mm}$ ) où règne un bioclimat semiaride à hiver très froid, ces formations présteppiques s'encartent en étage montagnard - méditerranéen. (Gordo, 2021). Cela semble correspondre à la série montagnarde méditerra-néenne de Quercus ilex subsp. ballota (Desf.) Samp., définie au Maroc par Banabid (1982). La présence d'espèces: telles que Quercus ilex subsp. ballota, Fraxinus dimorpha, Juniperus phoenicea subsp. phoenicea, J. oxycedrus subsp. oxycedrus et Ephedra major permet de rapporter ce type d'unités de végétations à l'ordre des Ephedro majoris-Juniperetalia phoeniceae Quézel \& Barbéro (1986) de la classe des Quercetea ilicis BraunBlanquet (1947) (Tableau 1).

Rappelons que l'ancienne station située au Djebel Mégriss (Atlas tellien) se trouve ainsi en position nettement plus septentrionale que celle de $\mathrm{Dj}$. M'Zi. Cet écart de latitude semble être compensé par l'altitude de notre station (2075 m, Figure 2).

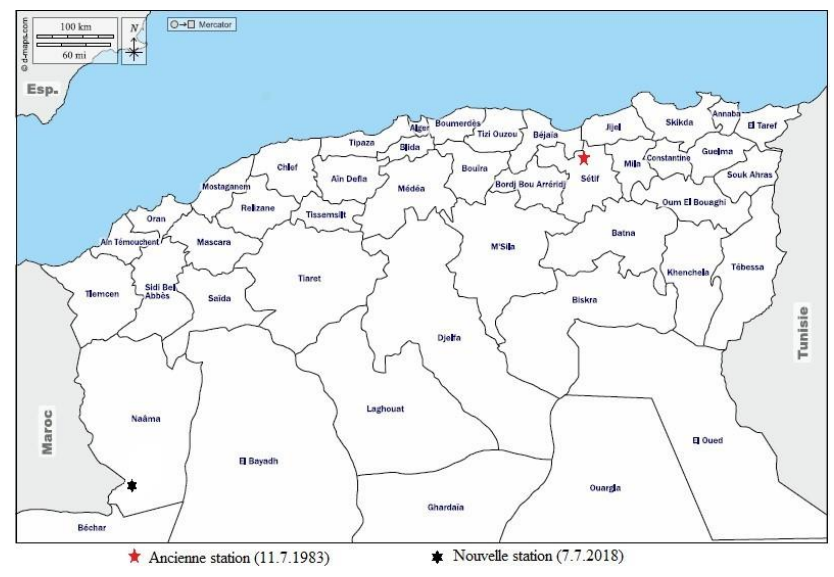

Figure 2. Localisation géographique de Stipa apertifolia subsp. longiglumis en Algérie.

Figure 2. Geographical location of Stipa apertifolia subsp. longiglumis in Algeria.

Plus au sud-est du secteur constantinois, précisément dans le $\mathrm{Dj}$. Chélia (Monts des Aurès), Trabut avait récolté $S$. atlantica $(=S$. pennata var. 
breviglumis Maire) endémique algérienne systématiquement très voisine de Stipa apertifolia subsp. longiglumis. Cela a été attesté par la planche «MPU010520 : Herb. L. Trabut, Algérie, Mont Chélia, Aurès, juillet 1892, L. Trabut,». étiquetée « S. pennata L.». D'après Smirnow (1929), l'holotype a été antérieurement collecté en 1853 par Munby dans la région de Dhalga (Algérie).

Tableau 1. Conditions stationnelles et cortège floristique de Stipa apertifolia subsp. longiglumis (H. Scholz) Vázquez \& Devesa au Djebel M'Zi (Aïn Sefra). (Nomenclature African Plant Database, 2021).

Table 1. Stationary conditions and floristic procession of Stipa apertifolia subsp. longiglumis (H. Scholz) Vázquez \& Devesa at M'Zi mountain (Aïn Sefra). (African Plant Database Nomenclature, 2021).

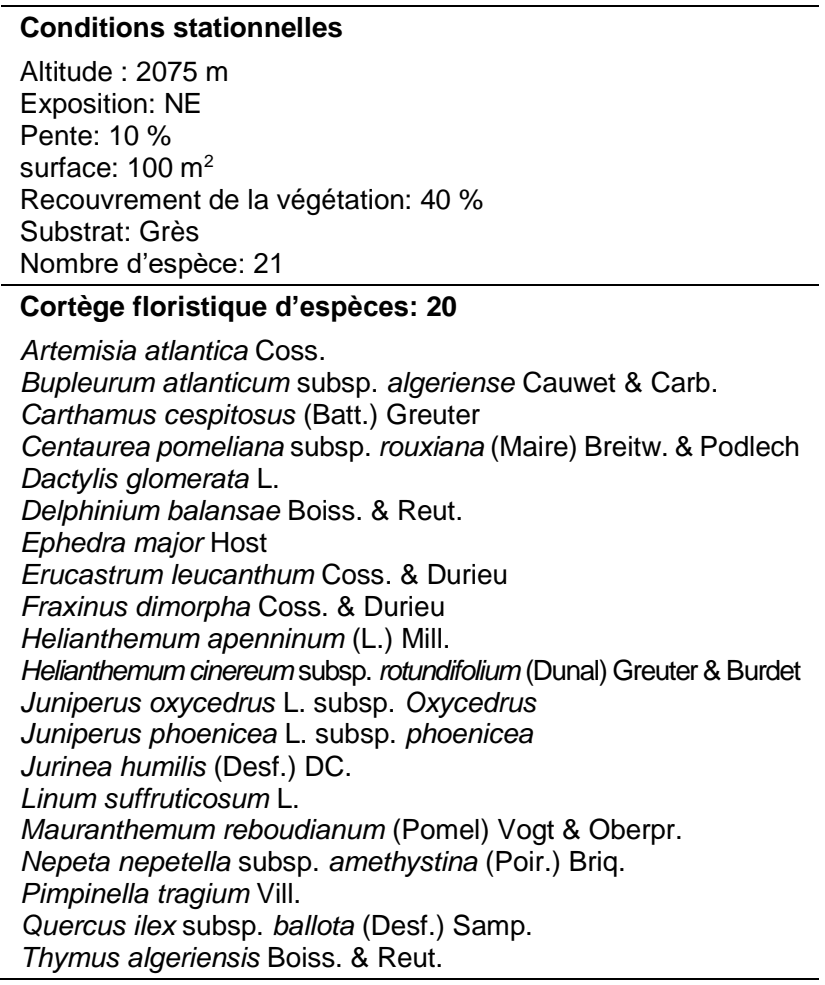

\section{Statut de conservation}

Bien qu'ayant été considéré comme rare par Quézel \& Santa (1962) dans les Aurès, Bellezma, Djurdjura et les Monts des Ksour, le taxon n'apparaît pas dans la liste des plantes non cultivées et protégées figurant dans le JORA (2012). De même, il ne fait pas partie de la liste rouge de l'UICN (2021). Sa répartition reste à préciser au Maroc comme tous les taxons appartenant à l'agrégat Stipa apertifolia d'après Fennane (2018).

Pour diagnostiquer l'état de conservation des espèces, il est d'usage de se servir des outils scientifiques et des critères définis par l'UICN (2018). Celle-ci préconise en premier lieu de commencer par une évaluation initiale, à condition que l'un des 5 (cinq) critères soit rempli au minimum. Pour attribuer un statut préliminaire à Stipa apertifolia subsp. longiglumis, nous avons tenu compte des données relatives à l'abondance des individus. Cela semble remplir le critère $B$ : aire de répartition est réduite. En effet, les vingt (20) individus matures et épars observés dans cette station relique sise aux pentes rocheuses du Djebel M'Zi occupent une superficie de $1800 \mathrm{~m}^{2}(0.0018 \mathrm{~km})$ environ. La surface de la zone d'occurrence, c'est-à-dire la superficie du Djebel M'Zi est estimée à $230 \mathrm{~km}^{2}$. Rappelons que seuls deux sujets paraissent dans notre relevé floristique. A partir de ces observations initiales, nous pouvons avancer que Stipa apertifolia subsp. longiglumis est en Danger Critique d'extinction (CR) suivant la classification de I'UICN (2018). Enfin, nous suggérons de faire figurer ce taxon dans la prochaine liste des plantes non cultivées et protégées au journal officiel d'Algérie.

\section{Remerciements}

Les auteurs tiennent à remercier Monsieur le professeur Mohamed Ibn Tattou (Univ. Mohamed V de Rabat) pour ses conseils judicieux.

\section{Bibliographie}

Aedo, C. (2020). Stipa L. In J.A. Devesa, C. Romero Zarco, A. Buira, A. Quintanar \& C. Aedo (Eds.), Flora Iberica. Vol. XIX (I), (pp. 78-100). Madrid: Real Jardín Botánico, CSIC.

African Plant Database (2021). Conservatoire et Jardin botaniques de la Ville de Genève and South African National Biodiversity Institute, Pretoria. Version 3.4.0. Published on the internet http:// www.ville-ge.ch/musinfo/bd/cjb/africa/ [Accessed April 10].

Benabid, A. (1982). Bref aperçu sur la zonation altitudinale de la végétation climacique du Maroc. In: Ecologia mediterranea, tome $8 n^{\circ} 1-2$. Définition et localisation des écosystèmes méditerranéens terrestres / Definition and localization of terrestrian Mediterranean biota. Saint-Maximin (France), (pp. 301-315). doi: https://doi.org/10.3406/ecmed.1982.1956

Benaissa, M. (2019). Étude de la dynamique de la diversité végétale des Monts des Ksour. Thèse Doctorat. Université Tlemcen. $187 \mathrm{p}+$ annexes.

JORA (2012). Décret exécutif du 18 janvier 2012 , portant la liste des espèces végétales non cultivées et protégées. Journal Officiel de République Algérienne, 3-12, 12-38.

Dobignard, A. \& Chatelain, C. (2010). Index synonymique de la flore d'Afrique du Nord. Conservatoire et Jardin Botaniques Genève.

El Oualidi, J., Khamar, H., Fennane, M., Ibn Tattou, M., Chauvet, S., \& Taleb, M.S. (2012). Checklist des endémiques et spécimens types de la flore vasculaire de l'Afrique du Nord. Document de I'Institut Scientifique, 25, Université Mohammed VAgdal, Rabat, Maroc.

Fennane, M. (2014). Stipa L. In M. Fennane et al. (eds), Flore Pratique du Maroc, 3, 513-318. Travaux de l'Institut Scientifique, Série Botanique, 40, Rabat.

Fennane, M. (2018). Éléments pour un Livre rouge de la flore vasculaire du Maroc, 10. Monocotyledonae (version 1). Montpellier : Tela-Botanica.

Gordo, B. \& Hadjadj-Aoul, S. (2019). L'endémisme floristique algéro-marocain dans les Monts des 
Ksour. Flora Mediterranea, 29, 129-142. doi: https://doi.org/10.7320/FIMedit29.129

Gordo, B. (2021). Analyse de la flore et de la végétation des Monts des Ksour. Thèse Doctorat. Université Oran 1 ABB. $190 \mathrm{p}+$ annexes.

Hochreutiner, BPG. (1904). Le sud oranais, étude floristique et phytogéographique. Conservatoire et Jardin Botaniques de la Ville de Genève.

Ibn Tattou, M. \& Fennane, M. (2008). Flore vasculaire du Maroc : Inventaire et chorologie, Asteraceae et Monocotylédones, 2. Travaux de l'Institut Scientifique, série Botanique, 39, Rabat, Maroc.

Maire, R. (1916). La végétation des montagnes du sud Oranais. Bulletin de la Société Botanique de Genève, 7, 210-292.

Maire, R. (1953). Flore de l'Afrique du Nord. Volume 2. Monocotyledonae. Paris: Le chevalier.

Quézel, P. \& Santa, S. (1962). Nouvelle flore de l'Algérie et des régions désertiques méridionales. Volume 1. C.N.R.S. Paris.
Sauvage, Ch. \& Vindt, J. (1949). Notes botaniques marocaines (Fasc. 1). Bulletin de la Société des Sciences Naturelles du Maroc, 29, 131-162.

Smirnow, P.A. (1929). Neue Stipen. Feddes Repertorium. Zeitschrift für Botanische Taxonomie und Geobotanik, 26, 264-271.

UICN (2018). Guide pratique pour la réalisation de listes rouges régionales des espèces menacéesMéthodologie de I'UICN \& démarche d'élaboration. Seconde édition. Paris.

UICN (2021). The IUCN Red List of Threatened Species. Version 2021-1. Published on the internet https:// www.iucnredlist.org. [Accessed April 10].

Vázquez, F. M. \& Devesa, J. A. (1996a). Floristic diversity of $\mathrm{N}$ Morocco. Notas sobre Stipa (Poaceae). Lagascalia, (18) 2, 322-324.

Vázquez, F. M. \& Devesa, J. A. (1996b). Revision del género Stipa L. y Nassella Desv. (Poaceae) en la Península Ibérica e Islas Baleares. Acta Botanica Malacitana, 21, 125-189.

doi:https://doi.org/10.24310/abm.v21i0.8674 